\title{
A Phase 2, Double-Blind, Placebo-Controlled Randomized Trial Assessing the Efficacy of ABT-436, a Novel VIb Receptor Antagonist, for Alcohol Dependence
}

\author{
Megan L Ryan*,', Daniel E Falk', Joanne B Fertig', Beatrice Rendenbach-Mueller², David A Katz', \\ Katherine A Tracy ${ }^{2}$, Eric C Strain ${ }^{3}$, Kelly E Dunn ${ }^{3}$, Kyle Kampman ${ }^{4}$, Elizabeth Mahoney ${ }^{4}$, Domenic A Ciraulo ${ }^{5}$, \\ Laurie Sickles-Colaneri ${ }^{5}$, Nassima Ait-Daoud ${ }^{6}$, Bankole A Johnson ${ }^{6}$, Janet Ransom ${ }^{7}$, Charles Scott ${ }^{7}$, \\ George F Koob' and Raye Z Litten' for the National Institute on Alcohol Abuse and Alcoholism Clinical \\ Investigations Group (NCIG) Study Group \\ 'Division of Medications Development, National Institute on Alcohol Abuse and Alcoholism, Bethesda, MD, USA; ${ }^{2}$ Neuroscience Development, \\ AbbVie, North Chicago, IL, USA; ${ }^{3}$ Johns Hopkins University School of Medicine, Baltimore, MD, USA; ${ }^{4}$ University of Pennsylvania, Treatment \\ Research Center, Philadelphia, PA, USA; ${ }^{5}$ Boston University School of Medicine, Boston, MA, USA; ${ }^{6}$ Department of Psychiatric Medicine, University \\ of Virginia, Charlottesville, VA, USA; ${ }^{7}$ Fast Track Drugs and Biologics, North Potomac, MD, USA
}

\begin{abstract}
Alcohol use disorder has been linked to dysregulation of the brain stress systems, producing a negative emotional state leading to chronic relapsing behavior. Vasopressin receptors appear to have a regulatory role in stress, anxiety, and alcohol. This study evaluated the novel compound, ABT-436, a VIb receptor antagonist, in alcohol-dependent participants in a I2-week clinical trial. Men and women ( $n=150$ ) who met criteria for DSM-IV alcohol dependence were recruited across four sites. Participants received double-blind ABT-436 or placebo, and a computerized behavioral intervention. ABT-436 was titrated to $800 \mathrm{mg} /$ day during weeks $2-12$. Although the primary outcome, percentage of heavy drinking days, was lower in participants receiving ABT-436 compared with placebo, this difference was not statistically significant (31.3 vs 37.6, respectively; $p=0.172 ; d=0.20$ ). However, participants receiving ABT-436 had significantly greater percentage of days abstinent than those receiving placebo ( 51.2 vs 41.6 , respectively; $p=0.037 ; d=0.31$ ). No significant differences were found between treatment groups on any other measures of drinking, alcohol craving, or alcohol-related consequences. Smokers receiving ABT-436 smoked significantly fewer cigarettes per week than those receiving placebo $(p=0.046)$. ABT-436 was well tolerated, with diarrhea (mildto-moderate severity) being the most common side effect. In subgroup analyses, participants with relatively higher baseline levels of stress responded better to ABT-436 than placebo on select drinking outcomes, suggesting there may be value in testing medications targeting the vasopressin receptor in high stress, alcohol-dependent patients.

Neuropsychopharmacology (2017) 42, I012-1023; doi:10.1038/npp.2016.214; published online 19 October 2016
\end{abstract}

\section{INTRODUCTION}

Alcohol use disorder (AUD) is a devastating disease that is responsible for a myriad of medical, psychological, social, economic, and personal problems (Dawson et al, 2008; Roerecke and Rehm, 2014). A recent report indicates that $13.9 \%$ of US adults met the criteria for AUD during the past year, whereas $29.1 \%$ met AUD criteria at some time in their lives (Grant et al, 2015). The economic burden of alcohol misuse is staggering, costing the US more than $\$ 249$ billion in 2010 (Sacks et al, 2015). Fortunately, progress has been made in developing medications to treat AUD. This

* Correspondence: ML Ryan, Division of Medications Development, National Institute on Alcohol Abuse and Alcoholism, 5635 Fishers Lane, Room 205I, Bethesda, MD 20892-9304, USA, Tel: +30I 443 4225, E-mail: mryan I@mail.nih.gov

Received 27 April 2016; revised 30 June 2016; accepted 8 September 2016; accepted article preview online 23 September 2016 development parallels the progress in understanding the neurobiological basis of AUD development and maintenance. Four medications have been approved by the US Food and Drug Administration to treat alcohol dependence: disulfiram, oral naltrexone, long-acting injectable naltrexone, and acamprosate. Still, because of the heterogeneity of AUD, these medications are not effective for all people. Continued research is needed to develop additional medications that are effective and safe for a diverse AUD population, providing clinicians with a greater menu of options for treating this disorder.

To better understand AUD, researchers have conceptualized this complex disorder in terms of specific domains, including reward, incentive salience, negative emotionality, executive function, and social processes (Litten et al, 2015). These domains, in turn, can be linked to three stages of the addiction cycle: binge/intoxication, withdrawal/negative affect, and preoccupation/anticipation (Koob and Le Moal, 1997). It has been postulated that as the severity of AUD 
increases, there is an increased contribution of the withdrawal/negative affect stage and increased engagement of the brain stress systems, including the hypothalamic-pituitaryadrenal (HPA) axis and extrahypothalamic stress systems (Koob, 2008). Key neurotransmitters and their related receptors include corticotropin-releasing factor (CRF), norepinephrine, glucocorticoids, dynorphin/kappa opioid, hypocretin (orexin), and vasopressin. Medications that block the receptors of these brain stress systems successfully reduced excessive alcohol intake in animal models, particularly where the excessive drinking is linked to the alleviation of signs associated with withdrawal and protracted abstinence (negative reinforcement) (Koob and Mason, 2016).

Vasopressin (arginine vasopressin, or AVP), a nonapeptide synthesized in the hypothalamus, is one of two main neuropeptides responsible for regulating the HPA axis. Along with CRF, AVP stimulates the release of pituitary adrenocorticotropic hormone (ACTH) via the AVP 1b (V1b) receptor. The release of ACTH stimulates production of cortisol via the adrenal cortex and is central to the HPA axis response to stress (Carrasco and Van de Kar, 2003; Herman and Cullinan, 1997; Sapolsky et al, 2000; Tsigos and Chrousos, 2002). In addition, like CRF, extrahypothalamic vasopressin appears to play a significant role in regulating various complex behaviors in preclinical studies, including stress, anxiety-like behaviors, aggression, social affiliation, sexual pair bonding, and addiction (Caldwell et al, 2008).

When selectively blocked, the $\mathrm{V} 1 \mathrm{~b}$ receptor appears to modulate anxiety, depression, and addiction. Griebel et al, 2002 found that rats treated with V1b receptor antagonist SSR149415 displayed anxiolytic-like activity and antidepressant-like effects in models of anxiety and depression. In other preclinical studies in rats, V1b receptor antagonists blocked dependence-induced compulsive-like alcohol intake. For instance, Edwards et al, 2011 reported that V1b antagonist SSR149415 dose-dependently reduced alcohol intake in dependent rats and did not affect alcohol intake in nondependent rats. Zhou et al, 2011 reported that the V1b receptor antagonist SSR149415 significantly reduced alcohol intake in Sardinian alcohol-preferring rats, compared with non-preferring rats. It is important to note the consistency of the findings observed in these two studies conducted at independent labs and using different animal models. These findings provided a rationale for studying a $\mathrm{V} 1 \mathrm{~b}$ receptor antagonist for the treatment of AUDs.

The novel compound studied in this clinical trial, ABT-436, is a potent, full and competitive selective V1b receptor antagonist manufactured and provided by AbbVie. Phase 1 studies involving the safety, pharmacodynamics, and alcohol interaction for ABT-436 have been completed (Katz et al, 2016a,b). Here we report the results of a multi-site clinical trial to assess the efficacy and safety of ABT-436 in an alcohol-dependent population. Outcomes assessed during the 12-week trial included alcohol consumption, alcohol craving, and drinking consequences. Cigarette smoking was assessed because of the high comorbidity of cigarette smoking and alcohol consumption. Changes in mood were also assessed due to the impact on mood when the intake of these substances is modified.

\section{MATERIALS AND METHODS}

\section{Study Population}

Randomized participants $(n=150)$ were diagnosed with past year alcohol dependence according to the Diagnostic and Statistical Manual, 4th edition Text Revision (DSM-IV-TR) (American Psychiatric Association, 1994). Participants were eligible if they were 21 to 65 years of age, reported drinking an average of at least 28 standard drinks per week for women or 35 standard drinks per week for men during the 28-day period before consent, had a CIWA-AR score of $<10$, were not diagnosed with Axis I disorders (except panic disorder), do not have any underlying medical condition that could exacerbate during trial participation or could achieve plasma cortisol $\geqslant 18 \mu \mathrm{g} / \mathrm{dl}$ within $60 \mathrm{~min}$ after CORTROSYN challenge (ACTH stimulation test). Most psychiatric medications were excluded except for the stable use of antidepressants. (see Supplementary Appendices 1 and 2 for the full inclusion/exclusion criteria and assessment schedule, respectively).

\section{Study Design}

The study was a Phase 2, randomized, double-blind, placebocontrolled, parallel-group 12-week treatment trial. Candidates responded by telephone to advertisements at four academic sites in the US between 21 February 2013 and 21 October 2014. The study (Protocol NCIG 004) was approved by the local Institutional Review Board at each participating clinical site; all subjects participating in the study provided their written informed consent before initiation of any study procedures. See Supplementary Appendix 3 for details on clinical sites and study oversight.

In addition to screening and baseline visits, six in-clinic visits and six telephone visits were conducted. A follow-up telephone interview was conducted at week 15, 2 weeks after the last in-clinic study visit, to assess safety and determine any changes in drinking. Participants were required to have a breath alcohol concentration $\leqslant 0.02 \%$ to complete the in-clinic assessments.

Participants were randomly assigned, in a 1:1 ratio, to receive either ABT-436 or matched placebo using a permuted stratified block randomization procedure. The stratification variables were clinical site and Spielberger Trait Anxiety Index (STAI) score $(<39$ and $\geqslant 39)$ (Spielberger et al, 1983), the latter was selected to balance groups with respect to trait anxiety (George et al, 2008), which was hypothesized to influence treatment response. Randomization was implemented via a centralized, interactive web-based response system (IWRS). See Supplementary Appendix 4 for additional details on randomization and blinding.

\section{Investigational Product}

ABT-436 is a potent and selective $\mathrm{V} 1 \mathrm{~b}$ receptor antagonist developed and manufactured by AbbVie. Medication was dispensed at scheduled visits over 12 weeks using a doubleblind method. ABT-436 was supplied in $200 \mathrm{mg}$ tablets with identical matching placebo tablets. For both the ABT-436 and placebo groups, the amount was titrated from a starting dose of $200 \mathrm{mg}$ on day 1, and $200 \mathrm{mg}$ twice a day on days $2-7$, to a target dose of $400 \mathrm{mg}$, twice a day, on weeks 2-12. 
On the basis of studies conducted by AbbVie, the dose of 200-800 mg QD was the projected efficacious dose range based on data from preclinical efficacy models, pharmacokinetic/pharmacodynamic modeling for HPA axis attenuation (urine total glucocorticoids, urine-free cortisol, plasma ACTH, and serum cortisol), and an initial signal for efficacy in a Phase $1 \mathrm{~b}$ study in major depressive disorder patients with $800 \mathrm{mg}$ QD of ABT-436. Although single doses up to $1600 \mathrm{mg}$ and multiple doses up to $1500 \mathrm{mg}$ were generally well tolerated in Phase 1 studies in mostly male subjects, $400 \mathrm{mg}$ BID was selected for this study to reduce the risk of drop outs due to gastrointestinal effects. Participants who could not tolerate the target dose were permitted to gradually reduce their dose to $600 \mathrm{mg}$ daily, then $400 \mathrm{mg}$ daily, and finally to $200 \mathrm{mg}$ daily until the dosage was well tolerated. If $200 \mathrm{mg}$ daily was not tolerated, medication was discontinued but those participants were encouraged to remain in the study and participate in study assessments. Dosage compliance was verified by comparing the patient's self-report to pill count. Medication compliance was calculated as the total amount of medication taken, divided by the total amount prescribed during the maintenance phase of the study (weeks 2-12). To validate adherence, ABT-436 plasma levels were determined from samples collected at weeks 1, 3, 5, 7, 9, and 13 and validated using a 96-well salting-out assisted liquid/ liquid extraction and high performance liquid chromatography tandem mass spectroscopy method (Zhang et al, 2009). ABT-436 plasma levels were not intended as a mediator of outcome given the variability of sample collection post dosing.

\section{Behavioral Platform}

All participants were required to view Take Control-a computerized bibliotherapy platform (Litten et al, 2013).

\section{Measures of Efficacy}

Alcohol consumption was captured via the Time-Line Follow-Back and Form 90 interview methodology and procedures (Sobell and Sobell, 1992; Miller, 1992). The $a$ priori primary efficacy end point was percentage of heavy drinking days measured weekly during the maintenance phase of the study (weeks 2-12). A 'heavy drinking day' was defined as four or more drinks (women) or five or more drinks (men) per drinking day.

A priori secondary efficacy end points included other drinking measures (ie, drinks per day, drinks per drinking day, percentage of days abstinent, percentage of very heavy drinking days $(8+/ 10+$ drinks per drinking day for women and men, respectively), percentage of subjects with no heavy drinking days, and percentage of subjects abstinent), during weeks 2-12; as well as severity of alcohol craving (Penn Alcohol Craving Scale (PACS)) (Flannery et al, 1999), number of alcohol-related consequences (ImBIBe; a revised and abbreviated form of the DrInC (Litten et al, 2013; Miller, 1995; Werner et al, 2008)), and number of cigarettes smoked per day (past week).

\section{Safety Assessments}

Safety was assessed via vital signs, blood chemistries and hematology, urine drug tests, breath alcohol concentration, adverse events, concomitant medication use, electrocardiogram), alcohol withdrawal (Clinical Institute Withdrawal Assessment for Alcohol-revised (CIWA-Ar) (Sullivan et al, 1989)), and psychiatric measures, including suicidal ideation (Columbia Suicide Severity Rating Scale (Posner et al, 2011)) and the Profile of Moods States (McNair et al, 1992). ABT-436's effect on the HPA axis was assessed by an ACTH stimulation test (CORTROSYN challenge) at screening, week 5 , and the end of the study. This ACTH stimulation test was conducted as a safety measure to ensure that alcoholdependent patients, who might be expected to have an already blunted cortisol response (Wand and Dobs, 1991), would not be further compromised by the potential cortisol reducing effects of ABT-436 (Katz et al, 2016a). Adverse events were assessed in the clinic and during telephone interviews using the open-ended question: 'How have you been feeling since your last visit?'

\section{Statistical Analysis}

All baseline, safety, and efficacy analyses (except for the prespecified model examining the number of cigarettes smoked per week) were analyzed on a modified intention-to-treat (mITT) population that included all randomized participants who received at least one dose of medication $(n=144$; ABT-436 $=73$, placebo $=71$ ). For efficacy analyses, one additional placebo subject had no drinking data during the maintenance period and one ABT-436 subject was missing data on a baseline covariate, resulting in 142 analyzable participants $(\mathrm{ABT}-436=72$, placebo $=70)$. The smoking efficacy model included only participants who were smokers at baseline (ie, smoked at least one cigarette in the past week) $(n=49 ;$ ABT $-436=23$, placebo $=26)$.

Continuous outcomes measured at multiple time points were analyzed using a repeated-measures mixed-effects model. Least-square means (LSMEANs), standard errors (SEs), and 95\% confidence intervals (CIs) are presented for each treatment group and were derived from fully adjusted models on untransformed outcomes (to facilitate clinical interpretation) averaged across the maintenance period (weeks 2-12). Cohen's $d$ and p-values were based on the fully adjusted models with the appropriately transformed outcome variables.

For the dichotomous drinking outcomes (ie, percentage of subjects abstinent and percentage of subjects with no heavy drinking days), unadjusted prevalence rates were determined. Odds ratios (ORs) and $p$-values were derived from unadjusted logistic regression models that included only the treatment group; covariates were not included because of insufficient numbers of abstinent and no heavy drinking events (Peduzzi et al, 1996).

Imputation for missing data generally was not performed; however, as a sensitivity analysis, missing data for the primary outcome, percentage of heavy drinking days, were handled in two ways: (a) by imputing missing data as heavy drinking days and (b) by using multiple imputation.

An exploratory analysis of five potential moderators was conducted on the primary efficacy outcome, percentage of heavy drinking days, and percentage of days abstinent to determine if a differential treatment effect existed during the maintenance period as a function of baseline characteristics. These characteristics included three measures of stress 
Table I Baseline Characteristics of Patients (mITT Population)

\begin{tabular}{|c|c|c|c|c|c|c|c|}
\hline & \multicolumn{3}{|c|}{ Placebo $(n=7 I)$} & \multicolumn{3}{|c|}{ ABT-436 $(n=73)$} & \multirow[t]{2}{*}{$P$-value ${ }^{a}$} \\
\hline & $n$ & Mean or \% & SD & $n$ & Mean or \% & SD & \\
\hline \multicolumn{8}{|l|}{ Demographics } \\
\hline Age & & 45.5 & 11.6 & & 45.8 & 10.2 & 0.857 \\
\hline Gender & & & & & & & 0.825 \\
\hline Male & 46 & $64.8 \%$ & & 46 & $63.0 \%$ & & \\
\hline Married & 32 & $45.1 \%$ & & 30 & $41.1 \%$ & & 0.630 \\
\hline Education (years) & & 14.3 & 2.9 & & 14.7 & 2.6 & 0.406 \\
\hline Race/Ethnicity & & & & & & & 0.832 \\
\hline White & 52 & $73.2 \%$ & & 52 & $71.2 \%$ & & \\
\hline Black & 15 & $21.1 \%$ & & 14 & $19.2 \%$ & & \\
\hline \multicolumn{8}{|l|}{ Self-reported alcohol consumption ${ }^{\text {b }}$} \\
\hline Drinks per day & & 10.1 & 5.4 & & 10.2 & 5.5 & 0.805 \\
\hline Drinks per drinking day & & 11.7 & 5.9 & & 12.3 & 6.9 & 0.579 \\
\hline Percent days abstinent & & 13.5 & 15.1 & & 14.7 & 18.2 & 0.963 \\
\hline Percent heavy drinking days & & 80.1 & 20.5 & & 78.8 & 21.4 & 0.697 \\
\hline Percent very heavy drinking days $(8+/ 10+)$ & & 46.5 & 33.3 & & 48.8 & 33.2 & 0.719 \\
\hline \multicolumn{8}{|l|}{ Other substance-related indicators } \\
\hline Penn Alcohol Craving Scale (PACS) score & & 16.4 & 5.6 & & 17.1 & 5.9 & 0.440 \\
\hline Alcohol-related consequences (ImBlBe) score & & 21.4 & 9.0 & & 20.5 & 8.3 & 0.530 \\
\hline Years of regular drinking & & 26.7 & 12.0 & & 26.8 & 10.7 & 0.980 \\
\hline Age of onset of regular drinking & & 18.8 & 6.4 & & 19.0 & 5.6 & 0.780 \\
\hline Copeptin (Pmol/L) & & 7.0 & 10.0 & & 6.7 & 4.9 & 0.843 \\
\hline \multicolumn{8}{|l|}{ Psychiatric characteristics } \\
\hline Spielberger Trait Anxiety Inventory (STAI) & & 39.4 & 10.8 & & 39.4 & 10.1 & 0.992 \\
\hline Spielberger Trait Anxiety Inventory (STAl) $>39$ & 38 & $53.5 \%$ & & 40 & $54.8 \%$ & & 0.878 \\
\hline Profile of Mood States (POMS)—Total Mood Disturbance score & & 10.6 & 23.2 & & 10.5 & 22.5 & 0.922 \\
\hline Clinical Institute Withdrawal Assessment of Alcohol—Revised (CIWA-AR) score & & 1.1 & 1.5 & & 1.0 & 1.5 & 0.516 \\
\hline
\end{tabular}

Abbreviations: $\mathrm{mITT}=$ modified intention-to-treat.

Note: Scale, number of questions (range), and interpretive values are as follows:

PACS: 5 questions $(0-30)$

ImBIBe: 15 questions (0-60).

ADS: 25 questions $(0-47)$.

POMS: 65 questions (-32-200).

STAl: 20 questions (20-69).

CIWA: 10 questions (0-67), > 10 indicative of alcohol withdrawal.

${ }^{a}$ Group mean differences were tested for significance via $t$-tests of independent samples for normally distributed variables or Wilcoxon rank-sum tests for skewed variables. Group prevalence rate differences were tested for significance via chi-square or Fisher's exact tests.

${ }^{b}$ Reflects mean values during the 28-day period (days I-28) before screening.

'Abstinence defined as abstinence (and never drink again) vs other. The denominator for the ABT-436 group includes 72 patients.

dMarijuana use based on positive urine drug screen. 
(STAI, POMS Tension-Anxiety subscale, and peak cortisol level in response to an ACTH stimulation test), gender, and alcohol consumption (drinks per day for the 28-day period before screening). A model similar to the primary efficacy model was used for each moderator; the moderator was included and the treatment-by-week interaction term was replaced with a treatment-by-moderator interaction term.

For all statistical tests, $p<0.05$ (two-tailed) was considered statistically significant. No adjustment was made for multiple inferential tests. For the primary outcome, an estimated sample size of 148 participants was required to obtain 126 study completers (63 per treatment group), yielding $80 \%$ power to detect a treatment effect (Cohen's $d=0.50$ ) with a two-tailed $t$-test at a 0.05 significance level. Data were analyzed with SAS version 9.2 (SAS Institute, Cary, NC). See Supplementary Appendix 5 for additional details regarding the statistical analysis.

\section{RESULTS}

\section{Study Sample}

Of the 415 participants consented for the study, 150 were randomized to receive $\mathrm{ABT}-436$ or a placebo $(n=75$ per group); 265 were excluded because they did not meet eligibility criteria or they chose not to participate (see Supplementary Appendix 6 for the CONSORT diagram). The top reasons for exclusion included: elevated blood pressure $(20.8 \%)$, positive urine toxicology drug screen (10.2\%), not meeting drinking criteria (8.3\%), and taking prohibited medication $(6.0 \%)$. The mITT population excluded six randomized participants who never received study medication. In the mITT population, more participants withdrew early from the study in the placebo than ABT-436 group (14 vs 10, respectively). Only one patient in the ABT-436 group withdrew from the study because of adverse events (AE) (diarrhea), compared with three in the placebo group (fogginess, rash, hives, and pruritus). However, more participants discontinued medication because of AEs (and stayed in the study) in the ABT-436 group than in the placebo group (8vs 0 , respectively).

Participants in the ABT-436 and placebo groups were not statistically different on any baseline characteristic (Table 1). Randomized participants were mostly male, white, employed, unmarried, and middle-aged. On average they drank heavily, consuming $\sim 10$ drinks per day, and met or exceeded four drinks (women) or five drinks (men) per drinking day on $\sim 79 \%$ of the days. With respect to treatment drinking goals, $\sim 13.2 \%$ desired permanent abstinence and to never drink again, whereas the majority sought to drink in a controlled manner. About one-third (34\%) smoked at least one cigarette in the week before the screening visit, averaging 98 cigarettes per week (among the smokers). Participants had very low levels of alcohol withdrawal (CIWA-Ar=1.1), near-normal levels of trait anxiety $(\mathrm{STAI}=39.4)$, and only modest levels of mood disturbance (POMS Total Mood Disturbance $=10.6$ ).

\section{Medication Compliance and Participation}

Overall medication compliance during the maintenance phase was $92.2 \%$ and was similar for both treatment groups
(92.1\% vs $92.3 \%$ for the placebo and ABT-436 groups, respectively; $p=0.916)$. The average daily dosage during the maintenance phase was nearly identical in both groups: $628 \mathrm{mg}$ in the placebo and $627 \mathrm{mg}$ in the ABT-436 groups (or 3.1 of the four maximum pills) $(p=0.980)$. Analyte levels of ABT-436 were largely consistent with patient self-reports of medication consumption (concordance rates: $78.5-89.7 \%$ during weeks $3,5,7$, and 9). The PK samples were taken to validate adherence and not intended as a mediator of outcome given that the samples were taken at different times post dosing. Overall, $84 \%$ of participants had complete drinking data during the maintenance phase, with the ABT-436 group being slightly higher than the placebo group (86.0\% vs $83.0 \%$, respectively), which was not statistically significant $(p=0.593)$.

\section{Primary Efficacy Outcome}

Averaged across the maintenance period (weeks 2-12), the ABT-436 group experienced somewhat lower adjusted levels of the primary outcome, percentage of heavy drinking days, than the placebo group (31.3 vs 37.6 , respectively; $\mathrm{d}=0.20)$, although this small effect was not statistically significant $(p=0.172)$ (Table 2). The treatment effect was statistically significant in the first week of the maintenance period (week 2) and was also largest in this week $(p=0.045$; $\mathrm{d}=0.34)$. However, across the weeks, the treatment effects were generally small (d's=0.03-0.34), did not reach statistical significance (with the exception of week 2), and did not differ statistically from each other (ie, treatment group by week interaction, $p=0.218$ ) (Figure 1a). The overall treatment effect was similar, and also not statistically significant, when missing drinking data were handled using multiple imputation - the adjusted mean percentage of heavy drinking days was 31.8 and 37.8 for ABT-436 and placebo, respectively $(p=0.175 ; \mathrm{d}=0.19)$. The treatment effect, however, increased slightly and achieved near-statistical significance with an imputation scheme that treated days with missing data as heavy drinking days (34.5 vs 43.8 for ABT-436 and placebo, respectively; $p=0.061 ; \mathrm{d}=0.26$ ). This small improvement in treatment effect was attributable to greater dropout (and more missing data to impute as heavy drinking days) in the placebo group than the ABT-436 group.

Treatment means suggest that ABT-436 appeared to have greater efficacy than placebo on the primary outcome, percentage of heavy drinking days, among participants with relatively greater baseline stress as measured by the POMS Tension-Anxiety, peak cortisol level during an ACTH stimulation test, and STAI (Figure 2); however, none of the five potential moderators were statistically significant (all treatment group by moderator interaction terms, $p$ 's $>0.152$ ). The treatment effect did not differ by gender or baseline drinks per day. The moderator results were similar using the outcome, percentage of days abstinent (see Supplementary Appendix 7).

\section{Secondary Efficacy Outcomes}

Averaged across the maintenance period, the ABT-436 group had significantly greater percentage of days abstinent than the placebo group (51.2 vs 41.6, respectively; $p=0.037$; 
Table 2 Treatment Outcomes: Differences Between Placebo and ABT-436 during Study Maintenance Phase (Weeks 2-12)

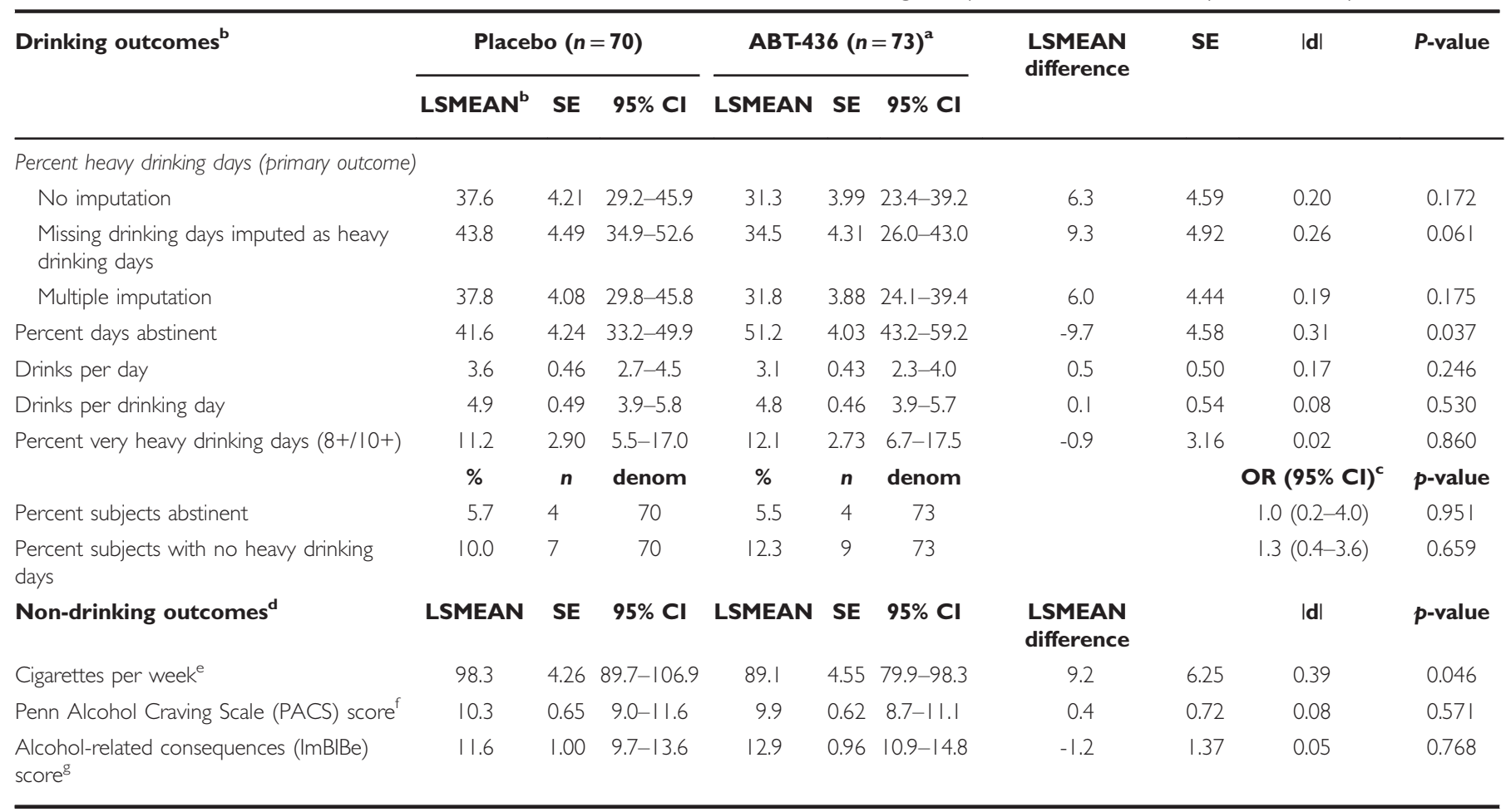

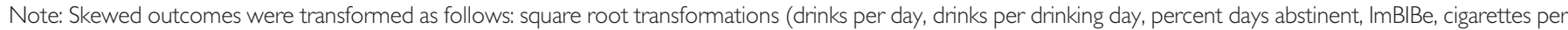
week, and POMS); inverse transformation (percent very heavy drinking days). No imputation was used for missing outcome data.

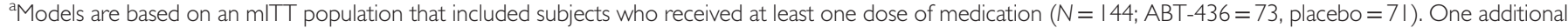
placebo subject had no drinking data during the maintenance period, and one ABT-436 subject was missing data on a baseline covariate, resulting in an analyzable $N=142(\mathrm{ABT}-436=72$, placebo $=70)$.

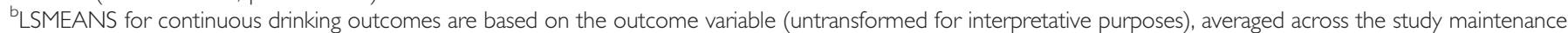

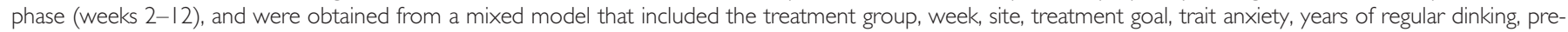

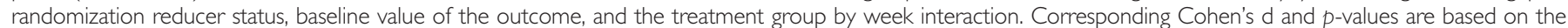
same model but with the appropriately transformed outcome variable.

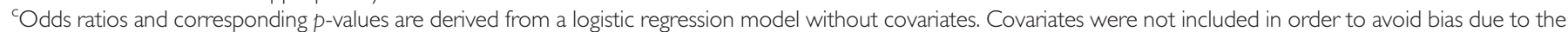
low number of events (Peduzzi et al, 1996).

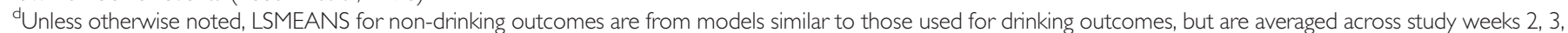
5, 7, 9, and 13; and only include treatment group, week, site, baseline value of outcome, and the treatment group by week interaction.

'The model for cigarettes per week included only patients who were smokers at baseline (ie, smoked at least one cigarette per day in the past week) ( $n=49$; $\mathrm{ABT}-436=23$, placebo $=26$ ).

'The model for PACS included additional covariates: treatment goal, alcohol dependence severity, and years of regular drinking.

sThe model for ImBIBe included an additional covariate: any prior alcohol treatment history (inpatient or outpatient) including AA attendance.

$\mathrm{d}=0.31$ ) (Table 2). This effect was largest in the first week and remained consistent throughout the maintenance period (Figure 1b). The treatment groups were not significantly different on any of the other five drinking outcomes.

No significant differences existed between the ABT-436 group and the placebo group on alcohol-related craving (PACS score; 9.9 vs 10.3 , respectively; $p=0.571 ; \mathrm{d}=0.08$ ) and consequences (ImBIBe score; 12.9 vs 11.6, respectively; $p=0.768 ; d=0.05)$. Among the non-drinking outcomes, averaged across the maintenance period, the smokers in the ABT-436 group smoked significantly fewer cigarettes per week (89.1 vs 98.3, respectively; $p=0.046 ; d=0.39$ ).

\section{Safety}

A total of $28 \mathrm{AE}$ categories were reported in at least $5 \%$ of participants from either treatment group (Table 3 ). Of these, diarrhea was significantly greater in the ABT-436 group than the placebo group ( $50.7 \%$ vs $19.7 \%$, respectively; $p<0.001)$. However, only four subjects stopped taking ABT-436 during the study as a result of gastrointestinal complaints. Although only a statistical trend, the ABT-436 group, compared with the placebo group, had greater rates of anxiety $(6.8 \%$ vs $0 \%$, respectively; $p=0.058)$ and nausea $(24.7 \%$ vs $12.7 \%$, respectively; $p=0.087)$. Among participants with these three $\mathrm{AE}$ categories, the majority experienced 'mild' symptoms (70\%), whereas the remaining participants experienced 'moderate' (25\%) and 'severe' symptoms (5\%). Three serious adverse events (SAEs) occurred during the treatment phase of the trial: cellulitis proximal to an insect sting (ABT-436), atrial fibrillation (ABT-436; occurring 9-weeks after discontinuing the study medication during the treatment period), and peptic ulcer disease (placebo). None of the serious adverse events were deemed related to the study 
a Percent Heavy Drinking Days (Primary Outcome)

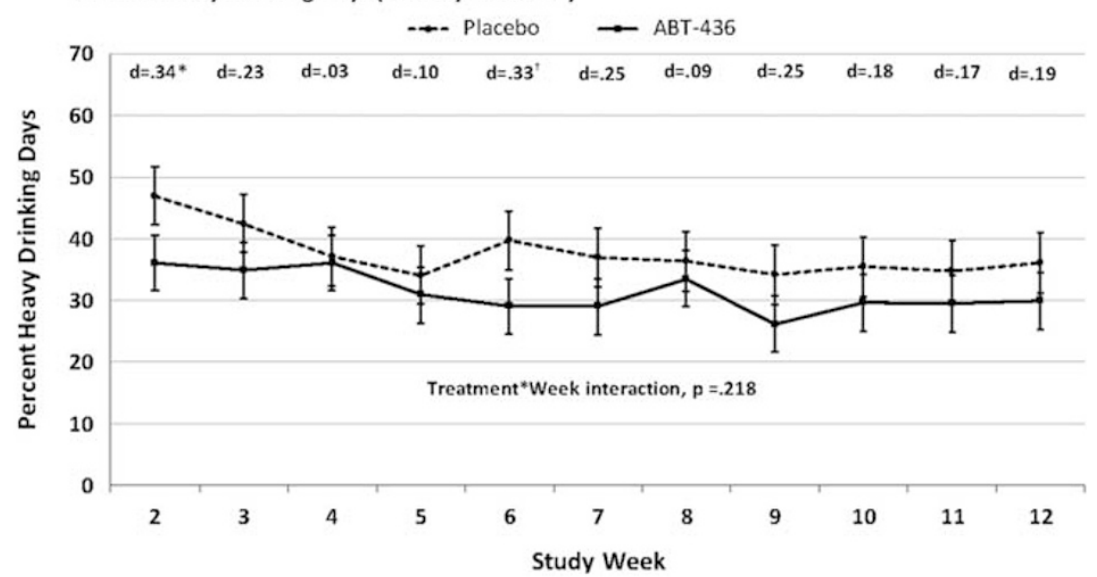

b Percent Days Abstinent

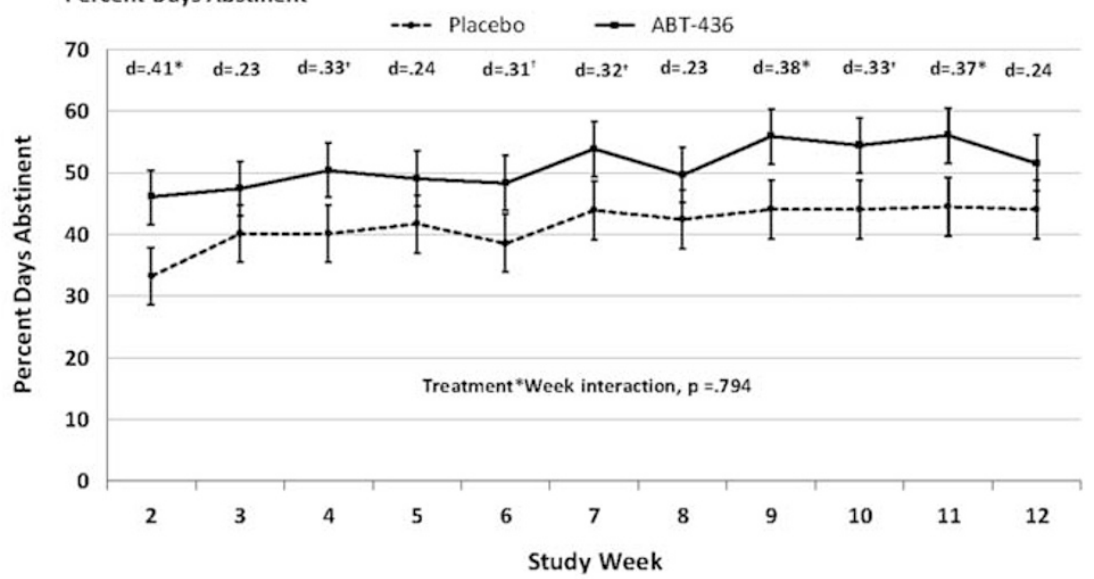

$\begin{array}{llllllllllll}\text { Number of Patients: } & & & & & & & & & \\ \text { Placebo: } & 70 & 67 & 65 & 64 & 61 & 60 & 59 & 59 & 59 & 59 & 59 \\ \text { ABT-436 } & 72 & 72 & 72 & 72 & 71 & 69 & 68 & 64 & 63 & 63 & 63\end{array}$

Figure I (a) Percent heavy drinking days and (b) Percent days abstinent. Means are LSMEANS obtained during the maintenance period (weeks 2-12) from a mixed model that includes treatment group, week, site, alcohol treatment goal, trait anxiety score, years drinking regularly, change in drinks per day between the screening and randomization, baseline equivalent of the outcome, and treatment group by week interaction. Error bars are standard errors. ${ }^{*} p<0.05 ;{ }^{\dagger} p<0.07$.

medication by the Medical Monitor. No clinically meaningful differences existed between ABT-436 and placebo on any other safety measures.

\section{DISCUSSION}

This multisite study evaluated the efficacy and safety of ABT-436, a vasopressin V1b receptor antagonist, in a mITT population of 144 alcohol-dependent participants. This is the first clinical trial of ABT-436 and the first multi-site trial to evaluate a vasopressin $\mathrm{V} 1 \mathrm{~b}$ receptor antagonist in an alcohol-dependent population. Participants receiving ABT-436 had significantly greater percentage of days abstinent than those receiving placebo (51.2 vs 41.6, respectively; $p=0.037 ; \mathrm{d}=0.31$ ). Although the primary outcome, percentage of heavy drinking days, was lower in participants receiving ABT-436 compared with placebo, this difference was not statistically significant (31.3 vs 37.6, respectively; $p=0.172 ; d=.20$ ). No significant differences were found between the two groups on any other measures of drinking, alcohol craving, or alcohol-related consequences.

It has been postulated that the release of vasopressin during alcohol withdrawal in alcohol-dependent individuals could foster a negative emotional state through stress sensitization, leading to an escalation in drinking (Edwards et al, 2011). Several lines of evidence support a relationship between vasopressin and drinking. For example, vasopressin and its receptors are highly expressed in the extended amygdala, an area important for stress sensitization and for stress-induced alcohol consumption (Koob, 2008). Increases in serum and urine vasopressin levels have been observed 
a

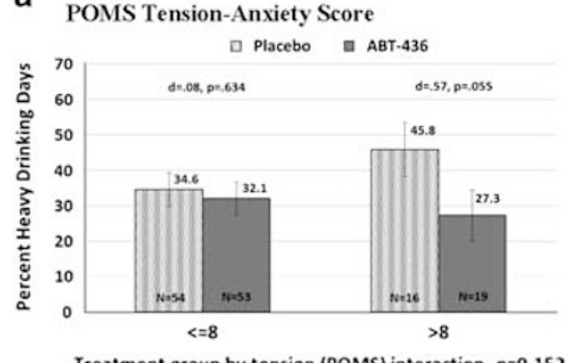

Treatment group by tension (POMS) interaction, $p=0.152$

d

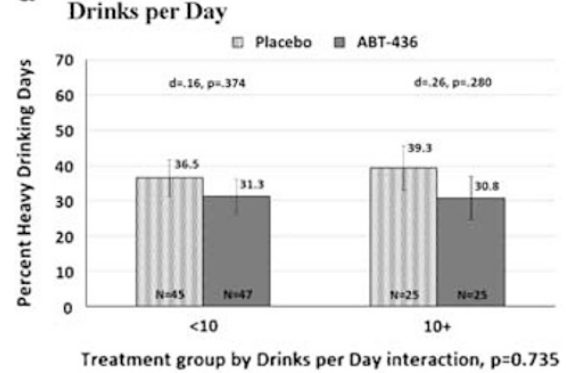

b

State Trait Anxiety Inventory (STAI) Score

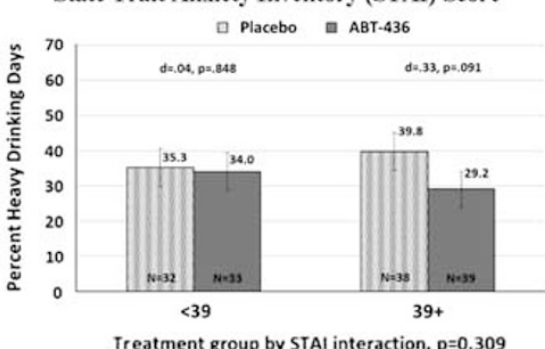

Treatment group by STAI interaction, $\mathrm{p}=0.309$

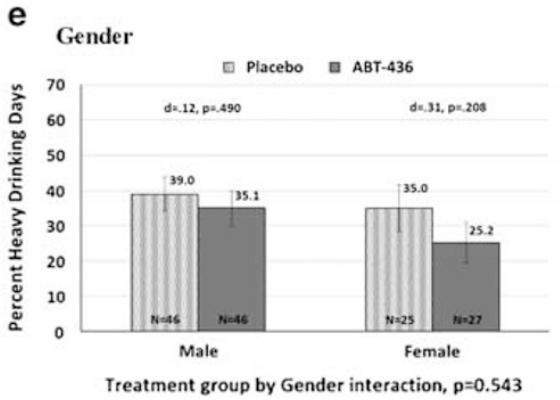
Peak Cortisol Level (from ACTH Stimulation Test)

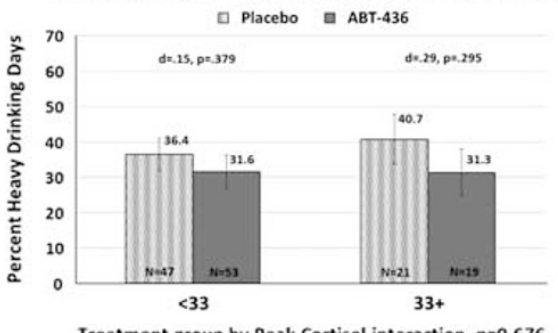

Treatment group by Peak Cortisol interaction, $p=0.676$

Figure 2 (a) POMS tension-anxiety score. Treatment group by tension (POMS) interaction, $p=0.152$. (b) State trait anxiety inventory (STAl) score. Treatment group by STAI interaction, $p=0.309$. (c) Peak cortisol level (from ACTH stimulation test). Treatment group by peak cortisol interaction, $p=0.676$. (d) Drinks per day. Treatment group by drinks per day interaction, $p=0.735$. (e) Gender. Treatment group by gender interaction, $p=0.543$.

during alcohol withdrawal, particularly when symptoms are severe (Eisenhofer et al, 1985; Trabert et al, 1992). Finally, Zhou et al, (2011) reported higher levels of basal vasopressin mRNA levels in the medial/central amygdala in Sardinian alcohol-preferring rats compared with Sardinian alcoholnon-preferring rats.

If ABT-436 works to attenuate the AVP/V1b-mediated stress response and, consequently, this leads to reduced drinking, then ABT-436 should theoretically have greater efficacy among individuals with relatively high levels of stress. Indeed, in this study, alcohol-dependent participants who experienced 'higher stress' at baseline appeared to respond better to $\mathrm{ABT}-436$ than those with fewer stress symptoms (Figure 2). Although the treatment-by-moderator interactions were not significant, the ABT-436 group had greater efficacy than placebo in subgroups with higher baseline trait anxiety (STAI), tension-anxiety (POMS), and peak cortisol in response to ACTH stimulation test. The latter, in particular, would be consistent with previous work suggesting that ABT-436's mechanism of action appears to be mediated by HPA axis reductions in serum ACTH and cortisol levels (Katz et al, 2016a). Compared with normative data, these subgroups had clinically significant levels of anxiety; the average STAI score in the elevated anxiety subgroup $($ mean $=47.4)$ was similar to that of adult neuropsychiatric patients diagnosed with anxiety (approximately $=48)$. In addition, the average POMS TensionAnxiety score in the elevated subgroup (mean $=12.4$ ) was almost one standard deviation above the normative adult mean (approximately $=8$ ) (Nyenhuis et al, 1999; Spielberger et al, 1983). However, despite the fact that ABT-436 had greater efficacy among participants with elevated stress symptoms, the compound did not produce a direct and measurable anxiolytic effect in these subgroups. For example, among those with elevated POMS Tension-Anxiety at baseline, the mean reduction in POMS Tension-Anxiety score from baseline to the end of treatment was not significantly different between the ABT-436 and placebo groups (4.7 and 5.2, respectively). Thus, although baseline anxiety may moderate the efficacy of ABT-436, the current study does not support a reduction in anxiety as the primary mediator/mechanism by which ABT-436 reduces drinking. It is possible that $\mathrm{ABT}-436$ reduced the stress response, compared with placebo, and it was simply not captured here. For example, ABT-436 may reduce drinking through stress systems that cannot be captured by standard anxiety tests. Future trials might maximize the treatment effect of ABT-436 by enriching the treatment population with participants who have clinically elevated anxiety (and/or hyper-reactivity to stress). In addition, progress toward understanding the mechanism of ABT-436 could be enhanced by developing better measures, which are sensitive and predictive to evaluate alcoholic's response to stress.

Although the mechanism of action appears to be related to the HPA axis, ABT-436 crosses the blood-brain barrier (AbbVie, data on file), and thus it also may act on extrahypothalamic areas, including the extended amygdala, an area involved in the brain stress system (Koob, 2008). It has recently been hypothesized that medications may need to normalize, rather than block, the activity of the HPA axis because of the blunted response of the HPA axis in chronic alcoholics (Adinoff et al, 2005). In addition, it has been postulated that chronic alcohol use drives the extrahypothalamic stress system increasing extrahypothalamic CRF as well as vasopressin to drive compulsive-like drinking (Vendruscolo et al, 2015). For example, evidence of a sensitized central amygdala includes a greater increase in CRF mRNA in alcohol-dependent rats than those observed 
Table 3 Number (\%) of Adverse Events Occurring in at Least 5\% of Patients in a Treatment Group ${ }^{\text {a }}$

\begin{tabular}{|c|c|c|c|c|c|}
\hline \multirow[t]{2}{*}{ MedDRA preferred term } & \multicolumn{2}{|c|}{$\begin{array}{l}\text { Placebo } \\
(n=7 I)\end{array}$} & \multicolumn{2}{|c|}{$\begin{array}{l}\text { ABT-436 } \\
(n=73)\end{array}$} & \multirow[t]{2}{*}{$p$-value ${ }^{b}$} \\
\hline & $n$ & $\%$ & $n$ & $\%$ & \\
\hline Diarrhea & 14 & $19.7 \%$ & 37 & $50.7 \%$ & $<0.001$ \\
\hline Headache & 18 & $25.4 \%$ & 17 & $23.3 \%$ & 0.847 \\
\hline Nausea & 9 & $12.7 \%$ & 18 & $24.7 \%$ & 0.087 \\
\hline Fatigue & 10 & $14.1 \%$ & 12 & $16.4 \%$ & 0.818 \\
\hline Nasopharyngitis & 5 & $7.0 \%$ & 8 & $11.0 \%$ & 0.563 \\
\hline Upper respiratory tract infection & 8 & $11.3 \%$ & 5 & $6.8 \%$ & 0.397 \\
\hline $\begin{array}{l}\text { Blood creatine phosphokinase } \\
\text { increased }\end{array}$ & 6 & $8.5 \%$ & 7 & $9.6 \%$ & 1.000 \\
\hline Vomiting & 5 & $7.0 \%$ & 7 & $9.6 \%$ & 0.765 \\
\hline Dizziness & 4 & $5.6 \%$ & 7 & $9.6 \%$ & 0.533 \\
\hline Back pain & 3 & $4.2 \%$ & 7 & $9.6 \%$ & 0.327 \\
\hline Dyspepsia & 4 & $5.6 \%$ & 5 & $6.8 \%$ & 1.000 \\
\hline Irritability & 4 & $5.6 \%$ & 5 & $6.8 \%$ & 1.000 \\
\hline Eosinophil count increased & 3 & $4.2 \%$ & 6 & $8.2 \%$ & 0.494 \\
\hline Arthralgia & 5 & $7.0 \%$ & 4 & $5.5 \%$ & 0.743 \\
\hline Disorientation & 4 & $5.6 \%$ & 4 & $5.5 \%$ & 1.000 \\
\hline Abdominal distension & 2 & $2.8 \%$ & 5 & $6.8 \%$ & 0.442 \\
\hline Abdominal pain & 2 & $2.8 \%$ & 5 & $6.8 \%$ & 0.442 \\
\hline Constipation & 4 & $5.6 \%$ & 3 & $4.1 \%$ & 0.717 \\
\hline Myalgia & 3 & $4.2 \%$ & 4 & $5.5 \%$ & 1.000 \\
\hline Rash & 4 & $5.6 \%$ & 3 & $4.1 \%$ & 0.717 \\
\hline Flatulence & 2 & $2.8 \%$ & 4 & $5.5 \%$ & 0.681 \\
\hline Rhinitis & I & $1.4 \%$ & 5 & $6.8 \%$ & 0.209 \\
\hline Neutrophil count increased & 4 & $5.6 \%$ & 2 & $2.7 \%$ & 0.438 \\
\hline Red blood cells urine positive & 2 & $2.8 \%$ & 4 & $5.5 \%$ & 0.681 \\
\hline Anxiety & 0 & $0.0 \%$ & 5 & $6.8 \%$ & 0.058 \\
\hline Pruritus & 4 & $5.6 \%$ & I & $1.4 \%$ & 0.206 \\
\hline Asthenia & 0 & $0.0 \%$ & 4 & $5.5 \%$ & 0.120 \\
\hline Decreased appetite & 0 & $0.0 \%$ & 4 & $5.5 \%$ & 0.120 \\
\hline
\end{tabular}

Note: bold are statistically significant $(p<0.05)$ or statistical trends $(p<0.10)$.

aultiple occurrences of a specific adverse event for a patient were counted once in the frequency for that adverse event.

${ }^{\text {b} G r o u p ~ p r e v a l e n c e ~ r a t e s ~ w e r e ~ t e s t e d ~ f o r ~ s i g n i f i c a n c e ~ v i a ~ c h i-s q u a r e ~ o r ~ F i s h e r ' s ~}$ exact tests.

in nondependent rats (Roberto et al, 2010). In addition, a CRF antagonist, D-Phe-CRF12-41 injected directly into the central nucleus of the amygdala, selectively decreased alcohol drinking in dependent but not nondependent rats (Funk et al, 2006). Similar results were observed in the same animal model of dependence with systemic administration of CRF-1 antagonists (Funk et al, 2006) and a V1b antagonist (Edwards et al, 2011).

It is interesting to note that, relative to placebo, ABT-436 significantly reduced the frequency of drinking episodes (as measured by percentage of days abstinent), but not the amount of drinking (drinks per day). The reason for this mixed effect on drinking pattern is unclear. Other medications, such as acamprosate, which, like ABT-436, are thought to impact the withdrawal/negative affect stage of drinking (DeWitte et al, 2005; Myrick et al, 2007), also have more of an effect on the frequency of drinking and less on total alcohol consumption. In contrast, medications like naltrexone, which influence the reward aspect of AUD (O'Malley et al, 2002; Davidson et al, 1996, 1999), have the opposite effect. For example, acamprosate increased the number of days abstinent in a clinical trial (Mason, 2005) but this medication did not reduce the number of drinks consumed after the initial drinking before human laboratory trials (Myrick et al, 2009; O'Malley et al, 2004). In contrast, naltrexone decreased the amount of alcohol consumed in human laboratory paradigms (Anton et al, 2004; O'Malley et al, 2002) and in clinical trials (particularly by decreasing the number of heavy drinking days), but had no impact on the frequency of drinking (Maisel et al, 2012). Thus, it is possible that the effect of ABT-436 on the withdrawal/ negative affect (stress) domain has more of an impact in reducing the frequency of drinking than in reducing total alcohol consumption.

In this trial, few subjects were able to achieve total abstinence or no heavy drinking days; moreover, the ABT-436 and placebo groups did not differ on rates of these outcomes. The fact that only a small minority of participants (13\%) expressed a goal of achieving total abstinence at baseline may have influenced this outcome. Also, participants were allowed to drink up to randomization and thus, may not have been able to make the drastic reduction in drinking necessary to achieve total abstinence by the end of the 1-week titration period. Future trials may be more likely to find an effect on abstinence and no heavy drinking outcomes by (a) enriching the study population with participants who have a goal of achieving these specific outcomes; (b) including a mandatory abstinence period prior randomization (eg, of at least 3 days); and (c) giving participants a longer period of time to change their drinking behavior before outcome assessment (grace period).

ABT-436 reduced the number of cigarettes per week among smokers. Drinking and smoking often co-occur, and both alcohol and nicotine can alter the physiological and subjective effects of each drug in terms of craving, reinforcement, and self-administration (reviewed by Britt and Bonci, 2013). As with drinking, stress-induced negative emotional states in animals can also drive smoking behavior (Koob and Le Moal, 2006; Leao et al, 2015). A recent review of biological mechanisms underlying the relationship between stress and smoking examined this relationship in humans (Richards et al, 2011). Although, the mechanism by which nicotinic receptors modulate drinking behaviors is not fully characterized, Leao et al, (2015) found that activation of nicotinic receptors facilitates the escalation of alcohol consumption suggesting that individual nicotinic receptor function individual differences may effect one's propensity for alcohol misuse. Indeed, stimulation of nicotinic receptors appear to have multiple effects on the brain, including modulation of areas involved in negative emotional states (reviewed by Wu et al, 2014). Thus, it is not surprising that medications like ABT-436, which are thought to reduce negative emotional states, could reduce smoking as well as alcohol consumption.

Because this was the first multisite clinical trial using ABT-436 in an alcohol-dependent population, the side-effect 
profile was closely monitored. ABT-436 was well tolerated, with generally mild-to-moderate side effects. The most common side effect was diarrhea, which occurred in $51 \%$ of the ABT-436-treated participants and $20 \%$ of the placebo group. However, only one participant receiving ABT-436 withdrew from the study because of this side effect. ABT-436 also did not have a clinically meaningful effect on the adrenal gland (ACTH stimulation test), mood, liver and renal function, and hematology and blood chemistry.

In summary, blocking the V1b receptors with ABT-436 reduced the frequency of drinking without significantly altering the amount consumed. Evidence exists that alcoholdependent participants with high stress levels responded better to ABT-436 in reducing both the frequency of drinking and heavy drinking days. Interestingly, ABT-436 was also efficacious in reducing smoking, perhaps by targeting a common withdrawal/stress domain underlying both drinking and smoking behaviors. Future studies exploring vasopressin and other targets within the stress system should focus on participants with high baseline stress levels because this subpopulation may be particularly responsive. This could effectively be accomplished in a human laboratory study testing the effects of ABT-436, as well as other anti-stress medications, on a validated paradigm of stress induction and stress-induced drinking. Co-occurring alcohol and tobacco use also should be considered when planning future studies, as medications targeting the stress system response may be beneficial in treating both addictions.

\section{FUNDING AND DISCLOSURE}

This study was supported by the National Institute on Alcohol Abuse and Alcoholism (NIAAA) (Contract HHSN2752014000011). Medication and matched placebo were provided by AbbVie. MLR, DEF, JBF, RZL, and GFK are Federal employees and declare no conflict of interest. $\mathrm{BR}-\mathrm{M}$ is an employee of AbbVie German $\mathrm{GmbH}$ and may own AbbVie stock or options. DAK received and continues to receive compensation from, and has majority stock ownership in, Sparrow Pharmaceuticals. DAK has been an employee of AbbVie, has received compensation from, and may own stock or options in, AbbVie, currently receives pension income from AbbVie, and has received compensation from NIAAA. KAT is currently an employee of Astellas Pharma and has been an employee of AbbVie and may own stock or options. ECS has received compensation from Johns Hopkins University School of Medicine, National Institute on Drug Abuse (NIDA), Egalet, Indivior Pharmaceuticals, Jazz Pharmaceuticals, The Oak Group, Pinney Associates, Relmada Therapeutics, and Zogenix. KED has received compensation from Johns Hopkins University and NIDA (grants). KK has received compensation from NIDA (grant), Indivior PLC (grant), Braeburn Pharmaceuticals (grant), Alkermes (grant), and Advisory Committee member Opiant Pharmaceuticals. EM has received compensation from the University of Pennsylvania. DAC has received compensation from the National Institute on Alcohol Abuse and Alcoholism (NIAAA), Boston University, Boston and Cape Alcohol and Addiction Medication Development Centers,
Massachusetts General Hospital, and Highpoint Treatment Center. LS-C has received compensation from the Boston University School of Medicine. NAD has received compensation from the University of Virginia and NIAAA (grant). BAJ has received compensation from the NIAAA, University of Virginia and University of Maryland School of Medicine; NIDA, NIH, and NIAAA (grants); Fast-Track Drugs and Biologics (contract); Eli Lilly, Organon, D\&A Pharma, ADial Pharmaceuticals, and Psychological Education Publishing Company (PEPCo) (consulting); holds patents for 'Serotonin transporter gene and treatment of alcoholism'; and holds equity ownership/stock options with ADial Pharmaceuticals (35\%), Life Foods (55\%), and PEPCo (100\%). JR and CS have received compensation from the NIAAA and Fast-Track Drugs and Biologics.

\section{ACKNOWLEDGMENTS}

The authors thank Barbara Vann of CSR for her excellent editorial comments.

\section{REFERENCES}

Adinoff B, Junghanns K, Kiefer F, Krishnan-Sarin S (2005). Suppression of the HPA axis stress-response: implications for relapse. Alcohol Clin Exp Res 29: 1351-1355.

American Psychiatric Association (1994). Diagnostic and Statistical Manual of Mental Disorders4th ednAmerican Psychiatric Publishing, Inc: Washington, D.C.

Anton RF, Drobes DJ, Voronin K, Durazo-Avizu R, Moak D (2004). Naltrexone effects on alcohol consumption in a clinical laboratory paradigm: temporal effects of drinking. Psychopharmacology 173: 32-40.

Britt JP, Bonci A (2013). Alcohol and tobacco: how smoking may promote excessive drinking. Neuron 79: 406-407.

Caldwell HK, Wersinger SR, Young WS (2008). The role of the vasopressin $1 \mathrm{~b}$ receptor in aggression and other social behaviours. Prog Brain Res 170: 65-72.

Carrasco GA, Van de Kar LD (2003). Neuroendocrine pharmacology of stress. Eur J Pharmacol 463: 235-272.

Davidson D, Palfai T, Bird C, Swift R (1999). Effects of naltrexone on alcohol self-administration in heavy drinkers. Alcohol Clin Exp Res 23: 195-203.

Davidson D, Swift R, Fitz E (1996). Naltrexone increases the latency to drink alcohol in social drinkers. Alcohol Clin Exp Res 20: 732-739.

Dawson DA, Li TK, Grant BF (2008). A prospective study of risk drinking: at risk for what? Drug Alcohol Depend 95: 62-72.

DeWitte P, Littleton J, Parot P, Koob G (2005). Neuroprotective and abstinence-promoting effects of acamprosate. CNS Drugs 19: 517-537.

Edwards S, Guerrero M, Ghoneim OM, Roberts E, Koob GF (2011). Evidence that vasopressin $\mathrm{V} 1 \mathrm{~b}$ receptors mediate the transition to excessive drinking in ethanol-dependent rats. Addict Biol 17: $76-85$.

Eisenhofer G, Lambie DG, Whiteside EA, Johnson RH (1985). Vasopressin concentrations during alcohol withdrawal. Br J Addict 80: 195-199.

Flannery B, Volpicelli J, Pettinati H. (1999). Psychometric properties of the penn alcohol craving scale. Alcohol Clin Exp Res 23: 1289-1295.

Funk CK, O’Dell LE, Crawford EF, Koob GF (2006). Corticotropinreleasing factor within the central nucleus of the amygdala medicates enhanced ethanol self-administration in withdrawn, ethanol-dependent rats. J Neurosci 26: 11324-11332. 
George DT, Gilman J, Hersh J, Thorsell A, Herion D, Geyer C et al (2008). Neurokinin 1 receptor antagonism as a possible therapy for alcoholism. Science 319: 1536-1539.

Grant BF, Goldstein RB, Saha TD, Chou SP, Jung J, Zhang $\mathrm{H}$ et al (2015). Epidemiology of DSM-5 alcohol use disorder: results from the National Epidemiologic Survey on Alcohol and Related Conditions III. JAMA Psychiatry 72: 757-766.

Griebel F, Simiand J, Serradeil-Le Gal C, Wagnon J, Pascal M, Scatton B et al (2002). Anxiolytic-and antidepressant-like effects of the non-peptide vasopressin $\mathrm{V} 1 \mathrm{~b}$ receptor antagonist, SSR149415, suggest an innovative approach for the treatment of stress-related disorders. PNAS 99: 6370-6375.

Herman JP, Cullinan WE (1997). Neurocircuitry of stress: central control of the hypothalamo-pituitary-adrenocortical axis. Trends Neurosci 20: 78-84.

Katz DA, Liu W, Locke C, Dutta S, Tracy KA (2016a). Clinical safety and hypothalamic-pituitary-adrenal axis effects of the arginine vasopressin type $1 \mathrm{~B}$ receptor antagonist ABT- 436 . Psychopharmacology 233: 71-81.

Katz DA, Locke C, Liu W, Zhang J, Achari R, Wesnes KA et al (2016b). Single dose interaction study of the arginine vasopressin type $1 \mathrm{~B}$ receptor antagonist $\mathrm{ABT}-436$ and alcohol in moderate alcohol drinkers. Alcohol Clin Exp Res 40: $838-845$.

Koob GF (2008). A role for brain stress systems in addiction. Neuron 59: 11-34.

Koob GF, Le Moal M (1997). Drug abuse: hedonic homeostatic dysregulation. Science 278: 52-58.

Koob GF, Le Moal M (2006). Neurobiology of Addiction. Elsevier Academic Press: Amsterdam.

Koob GF, Mason BJ (2016). Existing and future drugs for the treatment of the dark side of addiction. Annu Rev Pharmacol Toxicol 56: 299-322.

Leao RM, Cruz FC, Vendruscolo LF, de Guglielmo G, Logrip ML, Planeta CS et al (2015). Chronic nicotine activates stress/rewardrelated brain regions and facilitates the transition to compulsive alcohol drinking. J Neurosci 35: 6241-6253.

Litten RZ, Falk D, Ryan M, Fertig J (2015). Heterogeneity of alcohol use disorder: understanding mechanisms to advance personalized treatment. Alcohol Clin Exp Res 39: 579-584.

Litten RZ, Ryan ML, Fertig JB, Falk DE, Johnson B, Dunn KE et al (2013). A double-blind, placebo-controlled trial assessing the efficacy of varenicline tartrate for alcohol dependence. $J$ Addict Med 7: 277-286.

Maisel NC, Blodgett JC, Wilbourne PL, Humphreys K, Finney JW (2012). Meta-analysis of naltrexone and acamprosate for treating alcohol use disorders: when are these medications most helpful? Addiction 108: 275-293.

Mason BJ (2005). Acamprosate in the treatment of alcohol dependence. Expert Opin Pharmacother 6: 2103-2115.

McNair DM, Lorr M, Droppleman LF (1992). EdITs Manual for the Profile of Mood States. EdITs/Educational and Industrial Testing Service: San Diego, CA.

Miller W (1992). Form 90: A Structured Assessment Interview For Drinking And Related Behaviors (Test Manual). National Institute on Alcohol Abuse and Alcoholism: Bethesda, MD. (NIH publication no. 96-4004).

Miller W (1995). The drinker inventory of consequences (DrInC): an instrument for assessing adverse consequences of alcohol abuseInMattson M, Marshall LA(eds). NIAAA Project MATCH Monograph Series Vol. 4. National Institute on Alcohol Abuse and Alcoholism: Bethesda, MD. pp 1-94.

Myrick H, Anton R, Voronin K, Wang W, Henderson S (2007). A double-blind evaluation of gabapentin on alcohol effects and drinking in a clinical laboratory paradigm. Alcohol Clin Exp Res 31: 221-227.

Myrick H, Malcolm R, Randall PK, Boyle E, Anton RF, Becker HC et al (2009). A double-blind trial of gabapentin versus lorazepam in the treatment of alcohol withdrawal. Alcohol Clin Exp Res 33: $1582-1588$.

Nyenhuis DL, Yamamoto C, Luchetta T, Terrien A, Parmentier A (1999). Adult and geriatric normative data and validation of the profile of mood states. J Clin Psychol 55: 79-86.

O'Malley SS, Krishnan-Sarin S, Farren C, Sinha R, Kreek MJ (2002). Naltrexone decreases craving and alcohol self-administration in alcohol-dependent subjects and activates the hypothalamo-pituitary-adrenocortical axis. Psychopharmacology 160: 19-29.

O'Malley SS, McKee S, Krishnan-sarin S, Shi J, O'Connor P (2004). Abstinence effects and alcohol self-administration: acamprosate. Alcohol Clin Exp Res 28: 176A.

Peduzzi P, Concato J, Kemper E, Holford TR, Feinstein AR (1996). A simulation study of the number of events per variable in logistic regression analysis. J Clin Epidemiol 49: 1373-1379.

Posner K, Brown GK, Stanley B, Brent DA, Yershova KV, Oquendo MA et al (2011). The Columbia-suicide rating scale: initial validity and internal consistency findings from three multisite studies with adolescents and adults. Am J Psychiatry 168: 1266-1277.

Richards J, Stipelman BA, Bornovalova MA, Daughters S, Sinha R, Lejuez CW (2011). Biological mechanisms underlying the relationship between stress and smoking: state of the science and directions for future work. Biol Psychol 88: 1-12.

Roberto M, Cruz MT, Gilpin NW, Sabino V, Schweitzer P, Bajo M et al (2010). Corticotropin releasing factor-induced amygdala gamma-aminobutyric acid release plays a key role in alcohol dependence. Biol Psychiatry 67: 831-839.

Roerecke M, Rehm J (2014). Cause-specific mortality risk in alcohol use disorder treatment patients: a systematic review and metaanalysis. Int J Epidemiol 43: 906-916.

Sacks JJ, Gonzales KR, Bouchery EE, Tomedi LE, Brewer RD (2015). 2010 national and state costs of excessive alcohol consumption. Am J Prev Med 49: e73-e79.

Sapolsky RM, Romero LM, Munck AU (2000). How do glucocorticoids influence stress responses? Integrating permissive, suppressive, stimulatory, and preparative actions. Endocrine Rev 21: 55-89.

Sobell L, Sobell M (1992). Timeline follow-back: a technique for assessing self-reported alcohol consumption. In: Litten R, Allen J eds. Measuring Alcohol Consumption: Psychosocial and Biochemical Methods. Humana Press: Totowa, New Jersey. pp 41-72.

Spielberger CD, Gorsuch RL, Lushene R, Vagg PR, Jacobs GA. (1983). State-Trait Anxiety Inventory for Adults: Manual and Sample. Mind Garden, Inc, pp 1-82.

Sullivan JT, Sykora K, Schneiderman J, Naranjo CA, Sellers EM (1989). Assessment of alcohol withdrawal: the revised clinical institute withdrawal assessment for alcohol scale (CIWA-Ar). Br J Addict 84: 1353-1357.

Trabert W, Caspari D, Bernhard P, Biro G (1992). Inappropriate vasopressin secretion in severe alcohol withdrawal. Acta Psychiatr Scand 85: 376-379.

Tsigos C, Chrousos GP (2002). Hypothalamic-pituitary-adrenal axis, neuroendocrine factors and stress. J Psychosomatic Res 53: 865-871.

Vendruscolo LF, Estey D, Goodell V, Macshane LG, Logrip ML, Schlosburg JE et al (2015). Glucocorticoid receptor antagonism decreases alcohol seeking in alcohol-dependent individuals. J Clinical Invest 125: 3193-3197.

Wand GS, Dobs AS (1991). Alterations in the hypothalamicpituitary-adrenal axis in actively drinking alcoholics. J Clin Endocrinol Metab. 72: 1290-1295.

Werner M, Rentz A, Frank L, Bowman L, Duhig A, Moss H. (2008). Participant consequence measures. Presented at the annual meeting of the Research Society on Alcoholism, Washington, DC. 
Wu J, Gao M, Taylor DH (2014). Neuronal nicotinic acetylcholine receptors are important targets for alcohol reward and dependence. Acta Pharmacol Sin 35: 311-315.

Zhang J, Wu H, Kim E, El-Shourbagy TA (2009). Salting-out assisted liquid/liquid extraction with acetonitrile: a new high throughput sample preparation technique for good laboratory practice bioanalysis using liquid chromatography-mass spectrometry. Biomed Chromatogr 23: 419-425.

Zhou Y, Colombo G, Carai MAM, Ho A, Gessa GL, Kreek MJ (2011). Involvement of arginine vasopressin and V1b receptor in alcohol drinking in Sardinian alcohol-preferring rats. Alcohol Clin Exp Res 35: 1876-1883.

Supplementary Information accompanies the paper on the Neuropsychopharmacology website (http://www.nature.com/npp) 